\title{
Patient's Cognitive Function and Attitudes towards Family Involvement in Cancer Treatment Decision Making: A Patient-Family Caregiver Dyadic Analysis
}

\author{
Dong Wook Shin, MD, MBA, DrPH ${ }^{1,2}$ \\ Juhee Cho, PhD, MA ${ }^{34,5}$ \\ Debra L. Roter, DrPH, MPH ${ }^{5}$ \\ So Young Kim, MD, $P h D^{6,7}$ \\ Jong Hyock Park, MD, MPH, PhD 6,8 \\ Hyung Kook Yang, MD ${ }^{6}$ \\ Hyun Woo Lee, MD ${ }^{9}$ \\ Sun-Seog Kweon, MD, PhD'10 \\ Yune Sik Kang, MD, PhD ${ }^{11}$ \\ Keeho Park, MD, PhD ${ }^{6}$
}

*A list author's affiliations appears at the end of the paper.

\begin{abstract}
Purpose
Older patient populations commonly have cognitive impairment, which might impact decisional capacity. We examined patients and family caregivers preferences for family involvement in treatment decision making assuming different level of cognitive impairment, and sought to explore the factors associated with the preferences and the degree to which patients and family members agree on preferences.
\end{abstract}

\section{Materials and Methods}

A total of 358 elderly cancer patient and caregiver dyads were recruited from the 11 cancer centers in Korea and were asked to express their preferences for family involvement in treatment decision making using hypothetical scenarios with three different levels of cognitive status (intact, mild impairment, and severe impairment).

\section{Results}

Both patients and family caregivers preferred greater family dominance in treatment decision making with the increasing the level of cognitive impairment (39.7\%, 60.9\%, and 86.6\% for patients and $45.0 \%, 66.2 \%$, and $89.7 \%$ for caregivers in each scenarios). Patient and family caregiver concordance in decisional control preference was small for all three scenarios (weighted $\mathrm{k}=0.32, \mathrm{k}=0.26$, and $\mathrm{k}=0.36$, respectively). Higher patient education was associated with preference for patient dominance in treatment decision in conditions of both mild and severe cognitive impairment. The association of higher patient education and patient-caregiver preference concordance was positive with intact cognition, while it was negative with severe cognitive impairment.

\section{Conclusion}

Decision control preferences were affected by hypothesized cognitive status of the patients. Findings from our study would be helpful to develop effective strategy for optimizing family involvement in cancer treatment decision in the context of deteriorating cognitive function of the patients.
Correspondence: Jong Hyock Park, MD, MPH, PhD College of Medicine/Graduate School of Health Science Business Convergence, Chungbuk National University, 1 Chungdae-ro, Seowon-gu, Cheongju 28644, Korea Tel: 82-43-261-2873

Fax: 82-43-261-3459

E-mail: jonghyock@gmail.com

Co-correspondence: Keeho Park, MD, PhD Cancer Policy Branch, National Cancer Center, Cancer Control and Policy, Graduate School of Cancer Science and Policy, 323 Ilsan-ro, Ilsandong-gu, Goyang 10408, Korea Tel: $82-31-920-2160$

Fax: 82-31-920-2949

E-mail: park.keeho@gmail.com

Received April 26, 2017

Accepted July 1, 2017

Published Online July 4, 2017

\section{Key words}

Neoplasms, Family involvement, Cognitive function, Treatment decision, Caregivers 


\section{Introduction}

The ability to engage in the process of medical decision making is contingent on a patient's capacity to understand and use medical information to make treatment decisions consistent with one's values and preferences [1]. While cognitive function is an important component of capacity, with the exception of end-of-life decisions [2,3], relatively little research has focused on how patients and family members approach treatment decisions under conditions of cognitive impairment.

Older patient populations commonly suffer some degree of cognitive impairment $(\mathrm{CI})$ as a result of the normal aging process and comorbid medical conditions, as well as the potentially transitory effects of dehydration, medication side effects, anxiety, and depression. These patients are also at high risk for a cancer diagnosis; studies report that between $24 \%$ and $38 \%$ of elderly cancer patients have CI $[4,5]$. This impairment is likely to diminish decision making capacity at diagnosis, and late effects of cancer treatment (e.g., brain irradiation or chemotherapy [6-8]), is likely to further affect subsequent medical decisions.

While cancer treatment decision making is challenging for everyone, elderly patients face the additional burden of choosing among treatment options with diminished cognitive capacity and their families struggle with how best to support and protect them. This study was designed to contribute to the small literature in this area by examining preferences and agreement for family involvement in treatment decision making from the perspective of patients and family caregivers when presented with treatment scenarios in which a patient has no, mild, or severe cognitive impairment. We also sought to explore the factors associated with preferences for patients' active participation in treatment decision making and the degree to which patients and family members agree on preferences.

\section{Materials and Methods}

\section{Study participants}

Cancer patients 60 years or older and their caregivers were eligible for the the 2014 Cancer Patient Experience (CaPE) Survey, a nationwide, multicenter survey of the experience of cancer patients and their caregivers. We recruited participants from the National Cancer Center and other 10 regional cancer centers in Korea. Patients accompanied by family caregivers in outpatient waiting areas were recruited by trained research assistants who explained the survey purpose and procedures. Inclusion criteria for patients were as follows: (1) age 60 or older, (2) diagnosis of stomach, lung, or colorectal cancer, (3) currently receiving cancer treatment or follow-up care, and (4) in sufficient physical and mental health to complete the study questionnaire as judged by the trained research assistants. Patients who were diagnosed with dementia were excluded from the study.

After patients agreed to participate in the survey, adult family caregivers (age $\geq 18$ ) accompanying the patient to their cancer visit were also asked to participate in the survey. Patients and family members were instructed to complete the questionnaires independently and shown to separate areas of the waiting room to avoid consultation. For this study, we used patient-caregiver linked data.

\section{Measures}

Patients and family members were asked to express their preferences for family involvement in cancer treatment decision making using hypothetical scenarios with three different levels of cognitive status (intact, mild impairment, and severe impairment). As in previous studies $[2,3,9,10]$, a modification of the Decision Control Preferences Scale [11] was used to examine patient and family decisional control preferences. The response categories included: 1 (the patient makes the treatment decision on his/her own); 2 (the patient makes the treatment decision after hearing the family's opinion); 3 (the family makes the treatment decision after hearing the patient's opinion); and 4 (the family makes the treatment decision on their own). A pilot test with 10 patient-family member dyads confirmed that the questions and response options were clearly understood by the subjects.

Response options were on a 4-point ordinal scale reflecting the theoretical position that decision-making is unlikely to be equally shared [12] and the tendency of Asian respondents to choose a middle response style [13]. Respondents were asked to respond to three versions of the following question: "What do you think the desirable level of family involvement is in deciding your (patient version) or the patient's (caregiver version) cancer treatment when you (patient version) or the patient (caregiver version) has ([first ending] intact cognition) ([second ending] mild cognitive impairment) ([third ending] severe cognitive impairment).

Socio-demographic and medical information was obtained from study participants and the medical records of the patients were reviewed to collect information on American Joint Committee on Cancer (AJCC) cancer stage, treatmens, and other medical information. 


\section{Statistical analyses}

Patient and family caregiver responses to the decision preference questions were arrayed and examined by Friedman test to determine if there is difference in distribution between the dyads and between the different hypothetical scenarios (intact vs. mild impairment and mild vs. severe impairment). Next, we evaluated patient-family caregiver agreement on decisional control preference when presented with scenarios for intact cognition, mild impairment, or severe impairment by calculating percent agreement and kappa scores. Cases with missing response of either patients or family caregiver were excluded from the denominator. We also explored the association between patient and family caregiver characteristics and decisional control preferences with multivariate logistic regression, by dichotomizing responses into active patient (patient lead) or active family (family lead) as was done in previous studies [2,9]. All data analyses were conducted using STATA ver. 14.0 (StataCorp., College Station, TX).

\section{Ethical statement}

The study was approved by the Institutional Review Board of National Cancer Center (IRB No. NCCNCS13787) and performed in accordance with the principles of the Declaration of Helsinki. Written informed consents were obtained.

\section{Results}

\section{Subject characteristics}

A total of 358 patient and caregiver dyads enrolled in the study and completed provided complete survey information. Patients were on average 71 years of age, and $62 \%$ were male and slightly less than one-third had at least a college education (Table 1). Of total, $42.5 \%$ and $38.5 \%$ were stomach and colorectal cancer patients and disease was staged as AJCC stage I $(42.5 \%)$, II $(17.3 \%)$, III $(35.8 \%)$, and IV $(1.7 \%)$. More than half of the patients $(54.2 \%)$ were diagnosed within 1 years of the survey date, and most received surgery $(85.2 \%)$. Family caregivers were predominantly spouses $(50.8 \%)$ and adult children $(46.7 \%)$ and two-thirds of patients lived with their family caregiver.

\section{Decision control preferences and cognitive status}

As displayed in Fig. 1, there were significant differences in decision control preferences across levels of cognitive impair-
Table 1. Characteristics of patient-caregiver dyads

\begin{tabular}{|c|c|}
\hline Patient characteristic & No. $(\%)(n=358)$ \\
\hline Age, mean $\pm S D(y r)$ & $71.1 \pm 6.2$ \\
\hline \multicolumn{2}{|l|}{ Sex } \\
\hline Male & $222(62.0)$ \\
\hline Female & $136(38.0)$ \\
\hline \multicolumn{2}{|l|}{ Educational status } \\
\hline High school or less $(<12 \mathrm{yr})$ & $248(69.3)$ \\
\hline College and above ( $\geq 12 \mathrm{yr}$ ) & $110(30.7)$ \\
\hline \multicolumn{2}{|l|}{ Income status } \\
\hline$<2$ million KRW & $263(73.5)$ \\
\hline$\geq 2$ million KRW & $79(22.1)$ \\
\hline Missing & $16(4.5)$ \\
\hline \multicolumn{2}{|l|}{ Health insurance status } \\
\hline National Health Insurance & $347(96.9)$ \\
\hline Medical aid & $9(2.5)$ \\
\hline Others & $2(0.6)$ \\
\hline \multicolumn{2}{|l|}{ Cancer type } \\
\hline Stomach & $150(42.5)$ \\
\hline Lung and bronchus & $68(19.0)$ \\
\hline Colorectal & $138(38.5)$ \\
\hline \multicolumn{2}{|l|}{ AJCC cancer stage } \\
\hline I & $162(45.3)$ \\
\hline II & $62(17.3)$ \\
\hline III & $128(35.8)$ \\
\hline IV & $6(1.7)$ \\
\hline Time since diagnosis, mean $\pm \mathrm{SD}(\mathrm{yr})$ & $1.1 \pm 0.6$ \\
\hline$<1 \mathrm{yr}$ & $194(54.2)$ \\
\hline $1-2 \mathrm{yr}$ & $153(42.7)$ \\
\hline$>2 \mathrm{yr}$ & $11(3.1)$ \\
\hline \multicolumn{2}{|l|}{ Treatment received } \\
\hline Surgery & $305(85.2)$ \\
\hline Chemotherapy & $175(49.3)$ \\
\hline Radiotherapy & $53(15.4)$ \\
\hline \multicolumn{2}{|l|}{ Caregiver characteristics } \\
\hline Age, mean \pm SD (yr) & $56.0 \pm 13.7$ \\
\hline \multicolumn{2}{|l|}{ Sex } \\
\hline Male & $119(33.2)$ \\
\hline Female & $239(66.8)$ \\
\hline \multicolumn{2}{|l|}{ Educational status } \\
\hline High school or less $(<12$ yr) & $242(67.7)$ \\
\hline College and above ( $\geq 12 \mathrm{yr}$ ) & $115(32.1)$ \\
\hline Missing & $1(0.3)$ \\
\hline \multicolumn{2}{|l|}{ Income status } \\
\hline$<2$ million KRW & $171(47.8)$ \\
\hline$\geq 2$ million KRW & $177(49.4)$ \\
\hline Missing & $10(2.8)$ \\
\hline
\end{tabular}

(Continued to the next page) 
Table 1. Continued

\begin{tabular}{lc} 
Patient characteristic & No. $(\%)(\mathrm{n}=358)$ \\
Relationship to the patients & \\
Spouse & $182(50.8)$ \\
Adult children & $167(46.7)$ \\
$\quad$ Son & $69(19.3)$ \\
Daughter & $63(17.6)$ \\
Daughter in law & $32(8.9)$ \\
$\quad$ Son in law & $3(0.8)$ \\
Brother /Sister & $9(2.5)$ \\
Caregiving duration & \\
Mean \pm SD & $1.7 \pm 2.8$ \\
$<1$ yr & $206(60.4)$ \\
$1-2$ yr & $100(29.3)$ \\
$>2$ yr & $35(10.3)$ \\
Missing & $17(4.4)$ \\
Living with the patients & \\
Yes & $226(63.1)$ \\
No & $131(36.6)$ \\
Missing & $1(0.3)$ \\
\hline
\end{tabular}

SD, standard deviation; KRW, Korean won; AJCC, American Joint Committee on Cancer. ment; moreover, these differences were largely consistent for both patients and caregivers. More specifically, when considering treatment decisions under circumstances of intact cognitive function, $35 \%$ patients $(n=125)$ and $36.0 \%$ caregivers $(n=129)$ preferred that patients lead with input from family members while patients were more likely than caregivers to prefer that decisions are patient directed $(25 \%$ $[\mathrm{n}=90]$ vs. $18 \%$ [ $\mathrm{n}=66])$ while caregivers were more likely to prefer that decisions were family lead with patient input than patients (39\% [n=139] vs. 30\% [n=108]). Under the condition of mild cognitive impairment, $45 \%(\mathrm{n}=162)$ of patients and $55 \%(n=196)$ of caregivers preferred the family lead decisions with patient input. Finally, under the condition of severe cognitive impairment, the majority of patients $(57 \%$ [n=207] and $49 \%$ [ $n=177]$ ) of caregivers preferred to have the family make treatment decisions. A sizeable minority of patients $(29 \%$, $\mathrm{n}=103)$ and caregivers $(40 \%, \mathrm{n}=144)$ thought that families should lead these decisions with patient input.

\section{Concordance among responses}

Percentage agreement between patients and caregivers in responses to each scenario were $46.5 \%, 49.0 \%$, and $60.8 \%$, respectively. The weighted kappa reflecting patient and family caregiver concordance in decisional control preference was small but statistically significant for all three scenarios $(\kappa=0.32, \kappa=0.26$, and $\kappa=0.36$, respectively) (Table 2$)$.
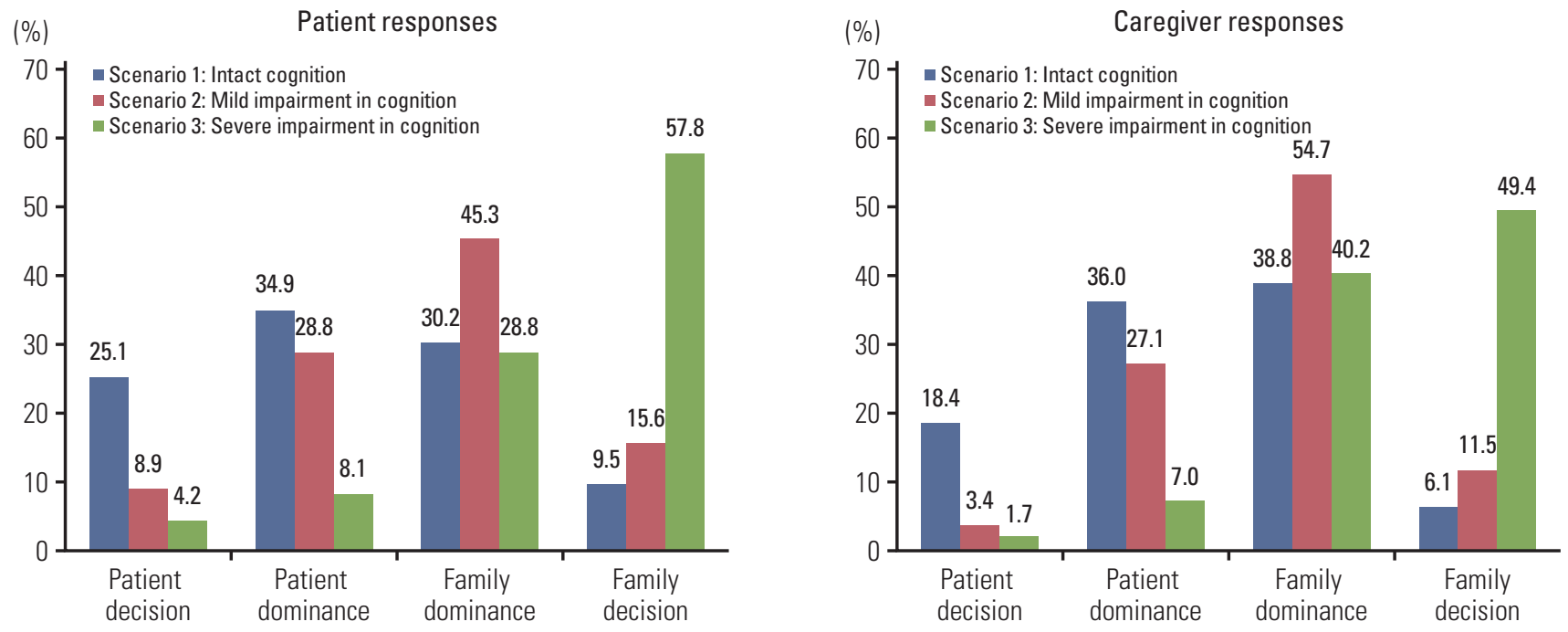

Fig. 1. Distribution of patient and caregiver responses to decision control preferences question by patients' hypothetical cognitive status. There was no significant difference in the level of decision control preference between patient and caregivers for all three scenarios $(p>0.05)$. There was significant difference in the level of decision control preference between scenarios (scenario 1 vs. scenario 2 and scenario 2 vs. scenario $3, \mathrm{p}<0.05$, respectively). 


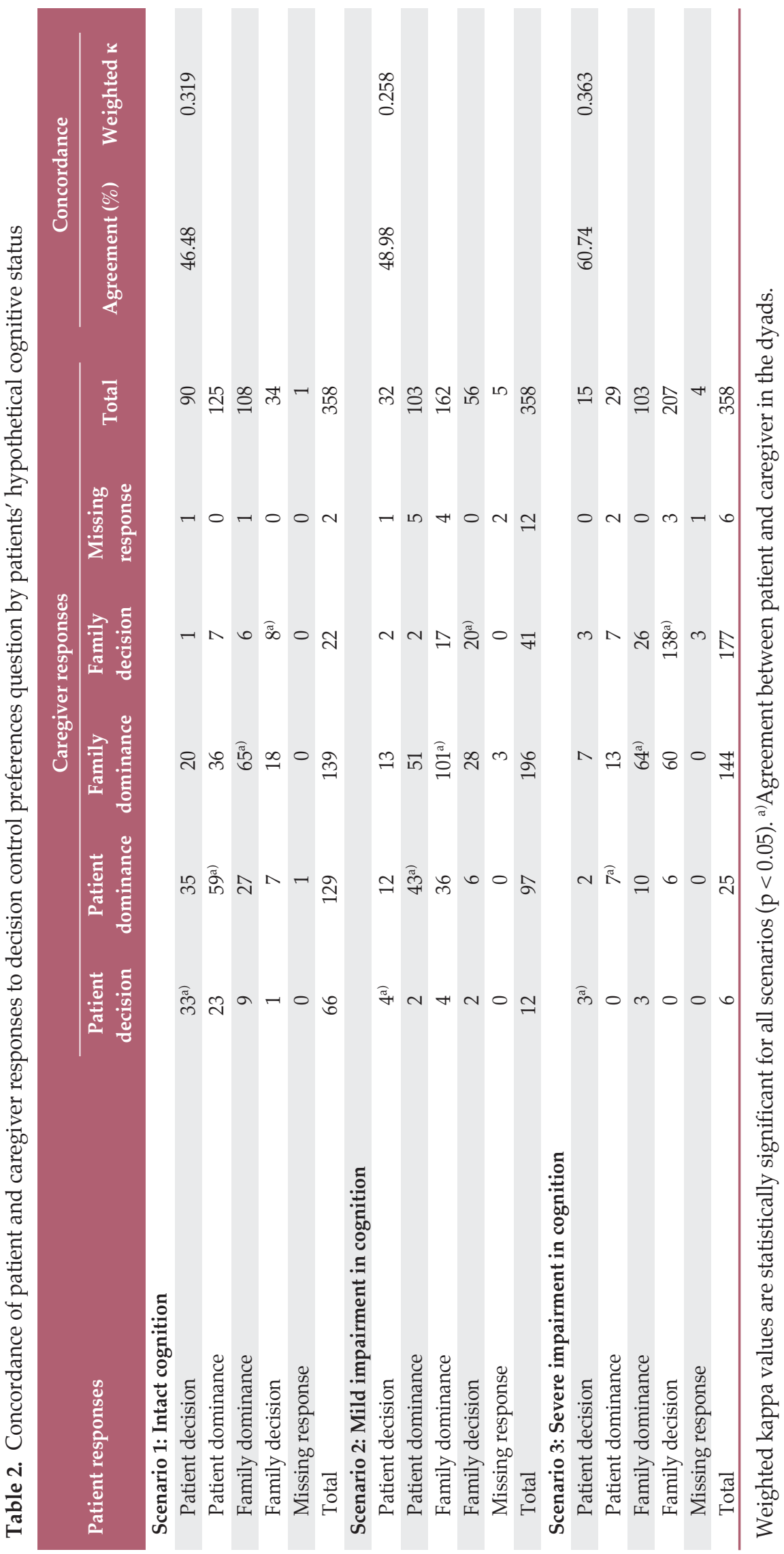




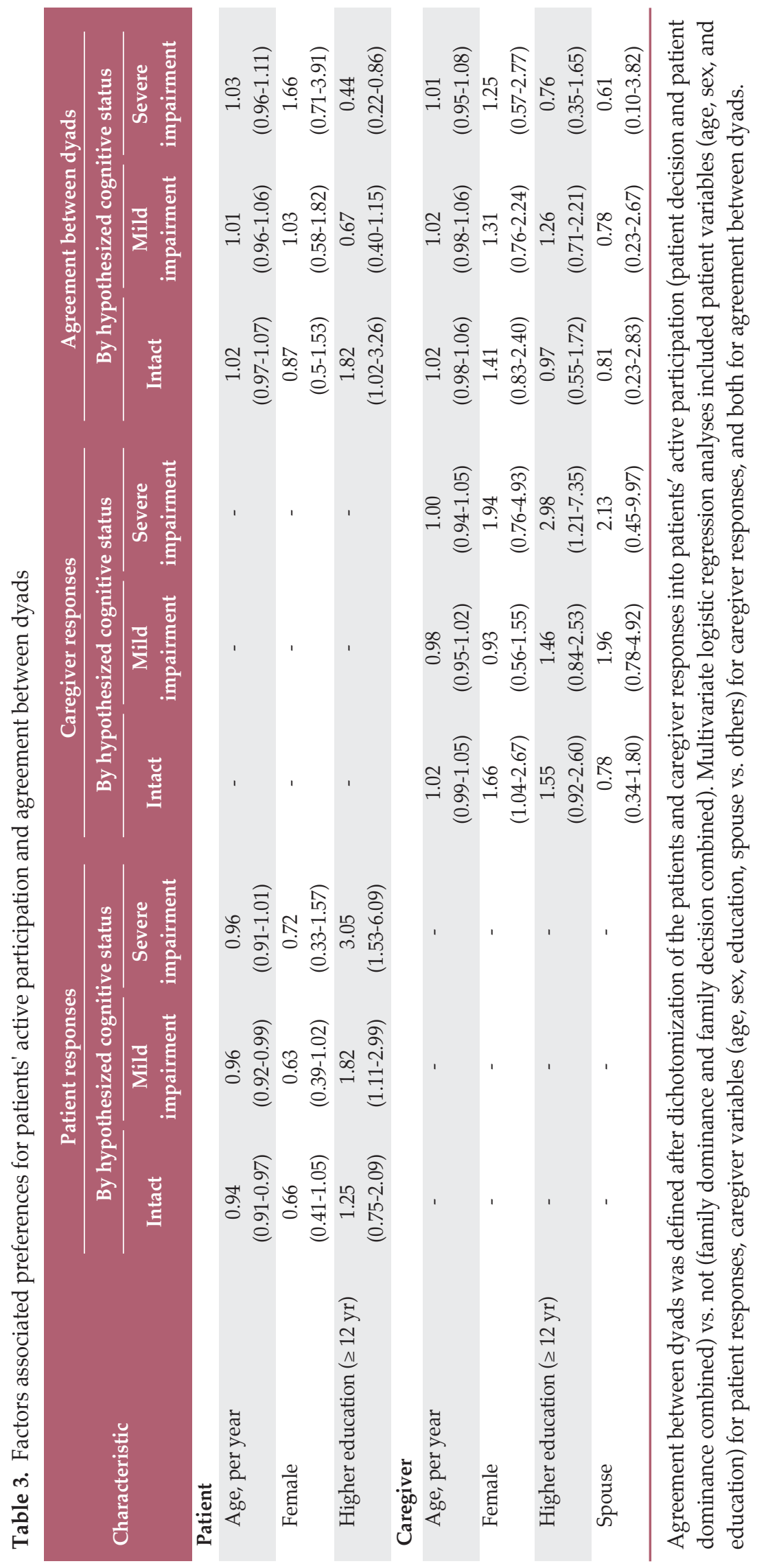




\section{Patient and Caregiver factors associated with decision control preferences}

As reflected in Table 3, older patients preferred less active participation in treatment decisions under conditions of intact cognition and mild impairment. Patients with higher levels of education preferred to dominate or lead decisions in contrast to less educated patients in conditions of both mild and severe CI with an odds ratio (OR) of 1.82 and 3.05, respectively.

Also displayed on Table 3 are two findings related to patient and caregiver agreement on decisional preferences. Higher education of patients was significantly associated patient-caregiver preference concordance when intact cognition was assumed; however, the association changed direction under conditions of severe $\mathrm{CI}$ such that the more highly educated the patient, the lower the agreement with caregivers regarding decisional preferences. Interestingly, more highly educated caregivers also preferred greater levels of patient decisional control under circumstances of severe impairment relative to less well educated caregivers (OR, 2.98).

\section{Discussion}

In this nationwide study of 358 cancer patients and their family caregivers, we systematically investigated preferences for family involvement in cancer treatment decision making when presented with scenarios across conditions of cognitive status. Under conditions of intact cognitive function, patients relative to caregivers were more likely to endorse patient directed decision making and less likely to endorse family led decisions. Both patients and family caregivers expressed a preference for greater family dominance in treatment decision making as the level of $\mathrm{CI}$ increased. While roughly half of patients and caregivers expressed a preference for the family to lead decisions, with patient input, under conditions of mild impairment, roughly $30 \%$ of both patients and caregivers preferred that patients lead decisions, with family input. In contrast, under the condition of severe impairment, about half of both patients and caregivers preferred that decisions be family directed; in this instance, however some $30 \%$ of patients and $40 \%$ of caregivers thought that family should lead the decision with patient input.

It seems quite natural that family take over the treatment decisions when $\mathrm{CI}$ is severe and likely to hinder decision making capacity [14], and is consistent with the findings from an observational study in which dementia family caregivers took a dominant or exclusive role in treatment deci- sions as cognitive function declined $[15,16]$.

Between $46 \%$ and $61 \%$ of patient and family dyads agreed on decision control preferences across scenarios, suggesting that a failure to agree is common and could be a source of family conflict, as has been observed in geriatric studies [17]. It is well known that family caregivers do not have an accurate sense of patients' values and preferences [1]; furthermore, the agreement rates found in this study are comparable to ultrasonographic findings of patient and family caregiver agreement on preferences for family involvement in decision making when considering conditions in which the patient is conscious (56\%) or unconscious (46\%) [2].

While patients appear to recognize the need for the family to lead treatment decisions under conditions of cognitive deterioration, many patients express a preference for continued involvement in making decisions about their own treatment and care $[1,18,19]$. Despite preferences to the contrary, actual involvement is often limited even at levels of mild impairment with family caregivers directing treatment decisions without patient input in consultation with physicians $[18,20]$. In some cases patients are excluded from participating in decisions because caregivers believe they are reducing patient burden and a source of distress [21], although a number of studies suggest that people with $\mathrm{CI}$ are usually able to communicate their values and preferences and can be active participants in treatment decisions [16,22]. Other studies suggest that some persons with dementia fear their future cognitive decline and subsequent loss of autonomy, and avoid involvement in treatment decisions [23,24], perhaps as a selfprotective mechanism [16].

Health care providers often rely on family members to make treatment and care decisions for patients with cognitive impairment, but current legal and ethical standards (e.g., The Mental Capacity Act 2005 in England and Wales) requires the safeguarding of the rights of people with limited capacity to make their own decisions for as long as possible [21].

Our study found that younger and more highly patients preferred greater decisional control, consistent with previous studies similarly finding that younger and better educated dementia patients remain involved in treatment decisions $[15,18]$. Female gender of caregiver was associated with preference for greater involvement of patients in treatment decisions, while male gender of the patients was negatively associated with it with marginal statistical significance. Such conflicting direction of influence of gender on treatment decisions involvement was also noted in previous studies $[18,25]$, and may reflect the traditional gender role in households in which most medical decisions are made by male [16]. Spouse caregivers showed some non-significant trends toward more active patient engagement in decision making, also consistent with previous studies with dementia [18,25]. Consistency with previous empirical studies, we believe that 
the hypothetical scenarios presented in the current study are likely to reflect actual practice.

One interesting finding of our study was that higher education of patients was associated with higher agreement in intact scenario, but changed to higher disagreement in CI scenarios. Patients with higher education tend to actively participate in the decision, and their wish agree with the family caregivers general willingness to involve them in treatment decisions [25]. However, patients want to remain involved in treatment decisions even when their cognitive function declines, while caregivers want to take a custodial role under this circumstance.

The clinical implications of our findings are that providers need to anticipate transitions in decisional preference among patients and their caregivers as cognitive status declines and that they may be able to support their patients and their families during this period in a variety of ways. First, screening and periodic assessment of CI using validated instruments to assess capacity, such as MacArthur Competency Assessment Tool for Treatment (MacCAT-T) [1,26], carries information that patients and their family caregivers want and need as they consider transitions in cognitive status and what that might mean for how treatment decisions are made. This is critically important since estimates of patients' decisional capacity only weakly correlates with objective assessment [1] and a determination of decisional capacity can achieve the proper balance between patient autonomy and best interest.

Second, clinicians can help patients and family caregivers to involve patients as much as possible by using simple language, repeating key points, asking targeted questions and obtaining verbal feedback about their understanding. Family caregiver can be also helped by information, emotional support, or communication skill training. Several interventions have been shown to be effective in improving the shared decision-making process among dyads of patients with dementia and their family caregivers [27-29].

Finally, the study findings indicate the need for regular assessments of patients' values and preferences to improve communication about treatment decisions within the patientcaregiver dyad from the early stages of cognitive impairment. CI in elderly cancer patients is usually progressive and can change rapidly due to complications from cancer or its treatment underscoring the need to discuss issues such as the goals of care, treatment preference, advance directives and surrogate decision makers while the patient still has capacity.

Several methodological limitations are noteworthy. First, the elderly cancer patients who were accompanied by family caregivers may be different in sociodemographic characteristics (e.g., age, education, and health status) than patients who chose not to participate or were not accompanied by a caregiver. These differences could influence their decisional control preferences. Secondly, we did not consider the issue of multiple caregivers who may share caregiving tasks and participate in treatment decisions. Third, our analytic framework was focused on dyadic relationship between patients and family caregivers, and did not consider the clinicians who are also important stakeholders in treatment decisions. Fourth, our study is based on scenarios and preferences expressed in response to hypothetical situations which may differ from those made under actual circumstances. Nevertheless, it is likely that the patients who show a preference for greater decisional involvement will be more involved in actual treatment decisions [30]. Moreover, all advance care planning needs to be based on the use of hypothetical situations by their very nature. Fifth, while our study is geographically nationwide, we could not determine representativeness of our sample as we could not collect information on the non-participants. However, participation rates in the CaPE surveys conducted in similar settings in previous years were estimated to be between $70 \%$ and $80 \%$. Finally, our study was conducted in South Korea, which has a tradition of Confucianism that individuals defer to the family in matters that consider the well-being of all members of a family. While a number of findings are consistent with those reported in Western studies, the generalizability across cultures should be further investigated.

Elderly patients now comprise the majority of cancer patient population in developed countries and are fast growing in developing countries. $\mathrm{CI}$ is common and may complicate treatment decision making process and threat patient autonomy. However, unfortunately, few guidelines are available for clinicians to guide treatment decision making of the elderly cancer patients with CI and their family caregivers. Findings from our study would be helpful to develop effective strategy for optimizing family involvement in cancer treatment decision in the context of deteriorating cognitive function of the patients.

\section{Conflicts of Interest}

Conflict of interest relevant to this article was not reported.

\section{Acknowledgments}

The following 11 Korean institutions participated in this study and data collection (in alphabetical order): National Cancer Center, Chonnam Regional Cancer Center, Chungbuk Regional Cancer Center, Daegu-Gyeongbuk Regional Cancer Center, Daejeon Regional Cancer Center, Gangwon Regional Cancer Center, Gyeonggi Regional Cancer Center, Gyeongnam Regional Cancer Center, Incheon Regional Cancer Center, Jeju Regional Cancer Center, and Ulsan Regional Cancer Center.

This work was supported by National Cancer Center (Grant No. 1610312). 


\section{Author Details}

${ }^{1}$ Supportive Care Center, Samsung Comprehensive Cancer Center, Seoul, ${ }^{2}$ Department of Family Medicine, Samsung Medical Center, Sungkyunkwan University School of Medicine, Seoul, ${ }^{3}$ Cancer Education Center, Samsung Comprehensive Cancer Center, School of Medicine and SAHIST, Sungkyunkwan University School of Medicine, Seoul, Korea, Departments of ${ }^{4}$ Epidemiology and ${ }^{5} \mathrm{Health}$, Behavior, and Society, Johns Hopkins Bloomberg School of Public Health, Baltimore, MD, USA, ${ }^{6}$ Cancer Policy Branch, National Can- cer Center, Goyang, 'Department of Public Health and Preventive Medicine, Chungbuk National University Hospital, Cheongju, ${ }^{8} \mathrm{Col}-$ lege of Medicine/Graduate School of Health Science Business Convergence, Chungbuk National University, Cheongju, ${ }^{9}$ Department of Hematology-Oncology, Ajou University School of Medicine, Suwon, ${ }^{10}$ Department of Preventive Medicine, Chonnam National University Medical School, Gwangju, ${ }^{11}$ Department of Preventive Medicine, Gyeongsang National University School of Medicine, Gyeongsang Institute of Health Science, Jinju, Korea

\section{References}

1. Hamann J, Bronner K, Margull J, Mendel R, Diehl-Schmid J, Buhner M, et al. Patient participation in medical and social decisions in Alzheimer's disease. J Am Geriatr Soc. 2011;59: 2045-52.

2. Sharma RK, Hughes MT, Nolan MT, Tudor C, Kub J, Terry PB, et al. Family understanding of seriously-ill patient preferences for family involvement in healthcare decision making. J Gen Intern Med. 2011;26:881-6.

3. Nolan MT, Hughes M, Narendra DP, Sood JR, Terry PB, Astrow $\mathrm{AB}$, et al. When patients lack capacity: the roles that patients with terminal diagnoses would choose for their physicians and loved ones in making medical decisions. J Pain Symptom Manage. 2005;30:342-53.

4. Caillet P, Canoui-Poitrine F, Vouriot J, Berle M, Reinald N, Krypciak S, et al. Comprehensive geriatric assessment in the decision-making process in elderly patients with cancer: ELCAPA study. J Clin Oncol. 2011;29:3636-42.

5. Molina-Garrido MJ, Guillen-Ponce C. Development of a cancer-specific comprehensive geriatric assessment in a university hospital in Spain. Crit Rev Oncol Hematol. 2011;77:148-61.

6. Grosshans DR, Meyers CA, Allen PK, Davenport SD, Komaki $R$. Neurocognitive function in patients with small cell lung cancer: effect of prophylactic cranial irradiation. Cancer. 2008;112:589-95.

7. Schagen SB, Boogerd W, Muller MJ, Huinink WT, Moonen L, Meinhardt W, et al. Cognitive complaints and cognitive impairment following BEP chemotherapy in patients with testicular cancer. Acta Oncol. 2008;47:63-70.

8. Chen X, Zhu C, Li J, Qiu L, Zhang L, Yu F, et al. Dissociation of decision making under ambiguity and decision making under risk in breast cancer patients receiving adjuvant chemotherapy: a neuropsychological study. Brain Res. 2013; 1533:63-72.

9. Shin DW, Cho J, Roter DL, Kim SY, Sohn SK, Yoon MS, et al. Preferences for and experiences of family involvement in cancer treatment decision-making: patient-caregiver dyads study. Psychooncology. 2013;22:2624-31.

10. Shin DW, Cho J, Roter DL, Kim SY, Yang HK, Park K, et al. Attitudes toward family involvement in cancer treatment decision making: the perspectives of patients, family caregivers, and their oncologists. Psychooncology. 2017;26:770-8.

11. Degner LF, Sloan JA, Venkatesh P. The Control Preferences Scale. Can J Nurs Res. 1997;29:21-43.

12. Makoul G, Clayman ML. An integrative model of shared decision making in medical encounters. Patient Educ Couns. 2006;60:301-12.

13. Harzing AW, Brown M, Koster K, Zhao S. Response style differences in cross-national research. Manag Int Rev. 2012;52: 341-63.

14. Wolfs CA, de Vugt ME, Verkaaik M, Haufe M, Verkade PJ, Verhey FR, et al. Rational decision-making about treatment and care in dementia: a contradiction in terms? Patient Educ Couns. 2012;87:43-8.

15. Hirschman KB, Xie SX, Feudtner C, Karlawish JH. How does an Alzheimer's disease patient's role in medical decision making change over time? J Geriatr Psychiatry Neurol. 2004;17: 55-60.

16. Miller LM, Whitlatch CJ, Lyons KS. Shared decision-making in dementia: a review of patient and family carer involvement. Dementia (London). 2016;15:1141-57.

17. Ishikawa H, Roter DL, Yamazaki Y, Hashimoto H, Yano E. Patients' perceptions of visit companions' helpfulness during Japanese geriatric medical visits. Patient Educ Couns. 2006;61: 80-6.

18. Hirschman KB, Joyce CM, James BD, Xie SX, Casarett DJ, Karlawish JH. Would caregivers of Alzheimer disease patients involve their relative in a decision to use an AD-slowing medication? Am J Geriatr Psychiatry. 2005;13:1014-21.

19. Karel MJ, Moye J, Bank A, Azar AR. Three methods of assessing values for advance care planning: comparing persons with and without dementia. J Aging Health. 2007;19:123-51.

20. Karlawish JH, Casarett D, Propert KJ, James BD, Clark CM. Relationship between Alzheimer's disease severity and patient participation in decisions about their medical care. J Geriatr Psychiatry Neurol. 2002;15:68-72.

21. Samsi K, Manthorpe J. Everyday decision-making in dementia: findings from a longitudinal interview study of people with dementia and family carers. Int Psychogeriatr. 2013;25: 
949-61.

22. Feinberg LF, Whitlatch CJ. Are persons with cognitive impairment able to state consistent choices? Gerontologist. 2001;41: 374-82.

23. de Boer ME, Droes RM, Jonker C, Eefsting JA, Hertogh CM. Thoughts on the future: the perspectives of elderly people with early-stage Alzheimer's disease and the implications for advance care planning. AJOB Prim Res. 2012;3:14-22.

24. MacQuarrie CR. Experiences in early stage Alzheimer's disease: understanding the paradox of acceptance and denial. Aging Ment Health. 2005;9:430-41.

25. Hirschman KB, Joyce CM, James BD, Xie SX, Karlawish JH. Do Alzheimer's disease patients want to participate in a treatment decision, and would their caregivers let them? Gerontologist. 2005;45:381-8.

26. Grisso T, Appelbaum PS. The MacArthur Treatment Competence Study. III: Abilities of patients to consent to psychiatric and medical treatments. Law Hum Behav. 1995;19:149-74.

27. Hilgeman MM, Allen RS, Snow AL, Durkin DW, DeCoster J, Burgio LD. Preserving Identity and Planning for Advance Care (PIPAC): preliminary outcomes from a patient-centered intervention for individuals with mild dementia. Aging Ment Health. 2014;18:411-24.

28. Murphy J, Oliver T. The use of Talking Mats to support people with dementia and their carers to make decisions together. Health Soc Care Community. 2013;21:171-80.

29. Whitlatch CJ, Judge K, Zarit SH, Femia E. Dyadic intervention for family caregivers and care receivers in early-stage dementia. Gerontologist. 2006;46:688-94.

30. Tariman JD, Berry DL, Cochrane B, Doorenbos A, Schepp K. Preferred and actual participation roles during health care decision making in persons with cancer: a systematic review. Ann Oncol. 2010;21:1145-51. 